OPEN ACCESS

Edited by:

Emmanuel Pinteaux,

The University of Manchester,

United Kingdom

Reviewed by:

Nicolas K. Khattar,

University of Louisville, United States

Ozge Altintas Kadirhan,

Kirklareli University, Turkey

${ }^{*}$ Correspondence:

Zhiping Hu

zhipinghu@csu.edu.cn

Chunli Chen

168202083@csu.edu.cn

†These authors have contributed equally to this work and share first authorship

Specialty section:

This article was submitted to

Stroke,

a section of the journal

Frontiers in Neurology

Received: 25 March 2021

Accepted: 12 July 2021

Published: 03 September 2021

Citation:

HuX, Zhu Y, Zhou F, Peng C, Hu Z and Chen $C$ (2021) Efficacy of Melatonin in Animal Models of Subarachnoid Hemorrhage: A Systematic Review and Stratified Meta-Analysis. Front. Neurol. 12:685731. doi: 10.3389/fneur.2021.685731

\section{Efficacy of Melatonin in Animal Models of Subarachnoid Hemorrhage: A Systematic Review and Stratified Meta-Analysis}

\author{
Xiangyu $\mathrm{Hu}^{\dagger}$, Yuwei Zhu ${ }^{\dagger}$, Fangfang Zhou, Cuiying Peng, Zhiping Hu* and Chunli Chen* \\ Department of Neurology, Second Xiangya Hospital, Central South University, Changsha, China
}

Background and Purpose: Subarachnoid hemorrhage $(\mathrm{SAH})$ is a severe disease characterized by sudden headache, loss of consciousness, or focal neurological deficits. Melatonin has been reported as a potential neuroprotective agent of SAH. It provides protective effects through the anti-inflammatory effects or the autophagy pathway. Our systematic review aims to evaluate the efficacy of melatonin administration on experimental SAH animals and offer support for the future clinical trial design of the melatonin treatment following $\mathrm{SAH}$.

Methods: The following online databases were searched for experimentally controlled studies of the effect of melatonin on SAH models: PubMed, Web of Knowledge, Embase, and China National Knowledge Infrastructure (all until March 2021). The melatonin effect on the brain water content (BWC) and neurological score (NS) were compared between the treatment and control groups using the standardized mean difference (SMD).

Results: Our literature identified 160 possible articles, and most of them were excluded due to duplication $(n=69)$ and failure to meet the inclusion criteria $(n=56)$. After screening the remaining 35 articles in detail, we excluded half of them because of no relevant outcome measures ( $n=16)$, no relevant interventions $(n=3)$, review articles $(n=1)$, duplicated publications $(n=1)$, and studies on humans or cells $(n=2)$. Finally, this systematic review contained 12 studies between 2008 and 2018. All studies were written in English except for one study in Chinese, and all of them showed the effect of melatonin on BWC and NS in SAH models.

Conclusion: Our research shows that melatonin can significantly improve the behavior and pathological results of SAH animal models. However, due to the small number of studies included in this meta-analysis, the experimental design and experimental method limitations should be considered when interpreting the results. Significant clinical and animal studies are still required to evaluate whether melatonin can be used in the adjuvant treatment of clinical SAH patients.

Keywords: subarachnoid hemorrhage, melatonin, systematic review, meta-analysis, animal models 


\section{INTRODUCTION}

Although it only leads to $4.4 \%$ of all types of strokes, subarachnoid hemorrhage (SAH) is a severe disease with high mortality and morbidity (1). SAH is characterized by clinical features such as sudden headache, single or combined with vomiting, loss of consciousness, or focal neurological deficits (2). At present, the management and prevention of SAH are still challenging due to its intricate pathophysiological conditions (3). Rupture of intracranial aneurysms is a typical cause of SAH (4). Zero to $24 \mathrm{~h}$ after hemorrhage, early brain injury (EBI) is observed in focal (5), together with blood-brain barrier damage and vascular spasms (6). Further cellular changes include inflammation and autophagy. The released products mediate and persist inflammatory responses by danger-associated molecular patterns (DAMPs) (7). Activation of the MAPK (mitogenactivated protein kinase) and Keap1-Nrf2-ARE [(Kelch-like ECH-Associating protein 1) nuclear factor erythroid 2 related factor 2-antioxidant response element] pathways may take over part of the inflammatory damage mechanism $(8,9)$. The EBI following SAH can also be induced by the autophagy mechanism (10), with the activation of mitochondria and the downstream pathway (11).

Recently, melatonin (N-acetyl-5-methoxytryptamine) has been reported as a potential neuroprotective agent of SAH. Melatonin, which derives from tryptophan (12), was demonstrated to counterwork oxidative stress and assist in scavenging free radicals (13). In 2010, researchers raised concerns about the anti-inflammatory effects of melatonin, specifically those comprising the reduction of the pathological changes in the tissues, attenuation of the development of $\mathrm{O}_{2}$-induced hyperalgesia and blockage of cyclooxygenase-2 (COX-2), and inducible nitric oxide synthase (iNOS) induction (14). Additionally, melatonin provides protective effects through the autophagy pathway in the Senescence Accelerated MouseProne 8 (SAMP8) mice (15). It seems that melatonin plays an effective neuroprotective role in SAH management $(16,17)$. However, this argument is inconsistent (18). The current study aims to evaluate and validate the efficacy of melatonin administration on experimental SAH animals. The related factors of research design that could shape the results will also be analyzed. Further, this preclinical study may offer support for the future clinical trial design of the melatonin treatment following SAH.

\section{METHODS}

\section{Data Sources, Search Strategy, and Selection Criteria}

The following online databases were searched for experimentally controlled studies of the effect of melatonin on SAH models: PubMed, Web of Knowledge, Embase, and China National Knowledge Infrastructure (all until March 2021). The following search terms were used: (subarachnoid $\mathrm{h}(\mathrm{a})$ emorrhage OR SAH OR aneurysm) AND (melatonin OR N-acetyl-5methoxytryptamine OR melatonergic agent) NOT human NOT patient. The reference lists of the included studies were also searched. Studies that met the following selection criteria were included: (a) experimental SAH was induced; (b) melatonin was administered before or after the induction of SAH; (c) control animals were used; (d) no co-treatments were performed; (e) therapeutic effects of melatonin were assessed by brain water content or neurobehavioral outcome.

\section{Data Extraction}

Two authors (XiangYu Hu and YuWei Zhu) independently extracted data from studies according to the selection criteria based on animal species, gender, number, intervention (dose and time of melatonin treatment), anesthetic technique, SAH induction method, measured outcomes, assessment time, and methodological quality score. Disagreements were addressed by a discussion with a third author (ChunLi Chen). When the included studies used multiple groups to assess dose-response relationships, we extracted data from each group individually. Graphically presented data were measured by the GetData Graph Digitizer software (version 2.26). The animal quantity, mean score, or content and standard deviation (SD), or standard error of the mean (SEM) in the melatonin and control groups were collected. When neurological tests were managed at different time points, only the final point was included. When it was unclear whether SD or SEM was the measure of variance, data was extracted as the SD due to it being a more accurate estimate, according to the present meta-analysis.

\section{Quality Assessment}

The methodological quality was evaluated using the collaborative approach to meta-analysis and review of Animal Data from Experimental Studies (CAMARADES) 10-item checklist with minor modifications (19). One point was given for each of the following criteria: peer-reviewed publication, control of temperature, random allocation to treatment or control, blinded induction of hemorrhage, blinded assessment of outcome, use of anesthetic without marked intrinsic neuroprotective activity, appropriate animal model (aged, diabetic or hypertensive), sample size calculation, compliance with animal welfare regulations, and statement of potential conflict of interests.

\section{Data Analysis}

A statistical software package (Stata, version 12.0; StataCorp LP, College Station, TX, USA) was used to perform data analyses. The melatonin effect on the brain water content (BWC) and neurological score (NS) were compared between the treatment and control groups using the standardized mean difference (the difference in the effect of melatonin between the treatment and control groups was divided by the total SD). This allowed for comparisons to be made when various measure methods or animal species were being used. The DerSimonian-Laird random-effects model was adopted to comprehensively estimate the effect size (20). We conducted a stratified meta-analysis to clarify the impact of drug dose, time of administration, overall study quality score, method of SAH induction, animal species, and type of anesthetic used. Funnel plotting was used to detect publication bias, the asymmetry of which was evaluated using Egger's test and the trim-and-fill method (21). Statistical 


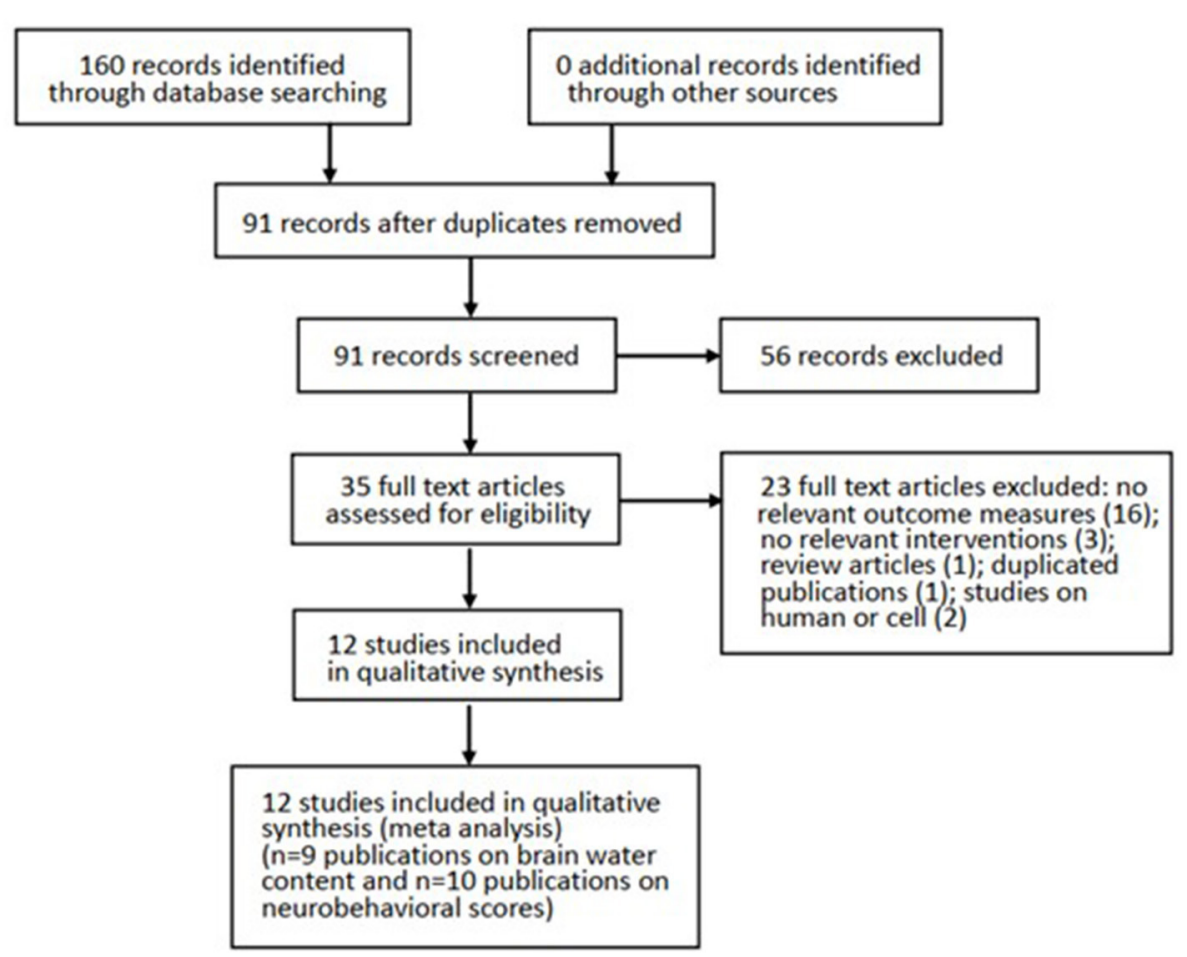

FIGURE 1 | Progression from literature search to meta-analysis. The number of exclusions from the initial literature search is shown.

significance was set at $P<0.05$ and the $95 \%$ confidence intervals (CIs) were calculated.

\section{RESULTS}

\section{Study Characteristics}

Our literature identified 160 possible articles, although most of them were excluded due to duplication $(n=69)$ and failure to meet the inclusion criteria $(n=56)$. After screening the remaining 35 articles in detail, we excluded half of them because of no relevant outcome measures $(n=16)$, no relevant interventions $(n=3)$, review articles $(n=1)$, duplicated publications $(n=1)$, and studies on humans or cells $(n=2)$. Finally, this systematic review contained 12 studies between 2008 and 2018. All studies were written in English except for one study in Chinese, and all of them showed the effect of melatonin on BWC and/or NS in SAH models (Figure 1).

We extracted data from 12 studies that describe BWC in 10 studies (26 comparisons) and NS in 12 studies (13 comparisons); the overall study characteristics are shown in Table 1. Among them, nine studies used SD rats, two studies used C57 mice, and one study used Wistar rats. Most of the studies involved male and adult models; only one study did not report the gender of animals. Regarding anesthesia, seven studies used pentobarbital, four studies used an anesthetic containing ketamine, and one study was done with isoflurane. All drugs were administered by intraperitoneal injection. Further, nine studies used endovascular perforation to induce the SAH model; the remaining three studies induced the model with autogenous blood injection. In most studies, the common initial drug dosage was $150 \mathrm{mg} / \mathrm{kg}$, but one study treated the rats with an initial drug dosage of $5 \mathrm{mg} / \mathrm{kg}$. Most studies performed the treatment of melatonin in $2 \mathrm{~h}$ after the induction of SAH while some included repeated injections. The melatonin treatment course was up to $72 \mathrm{~h}$. Assessments were performed 24-56 h after the induction of SAH.

\section{Study Quality}

Our systematic review included 12 studies in which the quality scores varied from three to seven. Among them, one study was assessed as a poor methodological quality study. Further, all the scores of others were higher than or equal to 4 ; their mean value (interquartile range) was 5.92 (1.5). All studies were peerreviewed publications. Nine (75\%) studies were completed with the control of temperature, the same number of studies allocated animals to treatment or control randomly, and 10 (83\%) studies used a blind assessment to evaluate outcomes.

For the assessment of BWC, the median number (interquartile range) of animals in the treatment and control groups was $6(0)$. For the assessment of NS, the median numbers (interquartile range) of animals in the treatment and control groups were 24 (15.5) and 21 (14), respectively. The details of the quality index are presented in Table 2 .

\section{Global Estimates of Efficacy}

The treatment of melatonin showed a significant reduction in the BWC by an SMD of -1.59 (95\% CI: $-2.02,-1.16$; $p<0.0001,10$ studies, 26 comparisons, Figure 2A), with a 
TABLE 1 | Design characteristics of included studies.

\begin{tabular}{|c|c|c|c|c|c|c|c|c|c|c|c|}
\hline References & $\begin{array}{l}\text { Animal, } \\
\text { sex }\end{array}$ & Age & Anesthetic & Route & $\begin{array}{l}\text { Method of } \\
\text { SAH }\end{array}$ & Initial dose & Total dose & $\begin{array}{l}\text { Time to } \\
\text { treatment }\end{array}$ & Tre $(n) / \operatorname{Con}(n)$ & Assessment time & $\begin{array}{l}\text { Outcome measure } \\
\text { (direction) }\end{array}$ \\
\hline Ayer et al. (22) & $\begin{array}{l}\text { SD rats, } \\
\text { Male }\end{array}$ & Adult & $\begin{array}{l}\text { Ketamine and } \\
\text { Xylazine }\end{array}$ & i.p. & $\begin{array}{l}\text { Endovascular } \\
\text { perforation }\end{array}$ & $150 \mathrm{mg} / \mathrm{kg}$ & $150 \mathrm{mg} / \mathrm{kg}$ & $2 \mathrm{~h}$ post SAH & 6,7 & $24 \mathrm{~h}$ & $\begin{array}{l}\text { BWC (lower is } \\
\text { better); }\end{array}$ \\
\hline Ersahin et al. (23) & $\begin{array}{l}\text { Wistar rats, } \\
\text { Male }\end{array}$ & Adult & $\begin{array}{l}\text { ketamine and } \\
\text { chlorpromazine }\end{array}$ & i.p. & $\begin{array}{l}\text { Autogenous } \\
\text { blood }\end{array}$ & 10 mg/kg & 30 mg/kg & $\begin{array}{l}\text { Immediately } \\
\text { post SAH (every } \\
24 \mathrm{~h} \text { for } 2 \text { days) }\end{array}$ & $6,66,6$ & $48 \mathrm{~h}$ & $\begin{array}{l}\text { BWC (lower } \\
\text { is better); NS (lower } \\
\text { is better) }\end{array}$ \\
\hline Wang et al. (24) & $\begin{array}{l}\text { SD rats, } \\
\text { Male }\end{array}$ & Adult & $\begin{array}{l}\text { ketamine and } \\
\text { xylazine }\end{array}$ & i.p. & $\begin{array}{l}\text { Autogenous } \\
\text { blood }\end{array}$ & 150 mg/kg & $300 \mathrm{mg} / \mathrm{kg}$ & $\begin{array}{l}2 \text { and } 24 h \text { post } \\
\text { SAH }\end{array}$ & 6,6 & $48 \mathrm{~h}$ & $\begin{array}{l}\text { BWC (lower is } \\
\text { better); }\end{array}$ \\
\hline Cai et al. (25) & $\begin{array}{l}\text { SD rats, } \\
\text { NR }\end{array}$ & Adult & $\begin{array}{l}\text { Ketamine and } \\
\text { xylazine }\end{array}$ & i.p. & $\begin{array}{l}\text { Autogenous } \\
\text { blood }\end{array}$ & 15 mg/kg & $60 \mathrm{mg} / \mathrm{kg}$ & $\begin{array}{l}5 \mathrm{~min}, 24,48, \\
\text { and } 72 \mathrm{~h} \text { post } \\
\text { SAH }\end{array}$ & 8,8 & $72 \mathrm{~h}$ & NS (higher is better) \\
\hline Chen et al. (26) & $\begin{array}{l}\text { SD rats, } \\
\text { Male }\end{array}$ & Adult & pentobarbital & i.p. & $\begin{array}{l}\text { Endovascular } \\
\text { perforation }\end{array}$ & $150 \mathrm{mg} / \mathrm{kg}$ & $150 \mathrm{mg} / \mathrm{kg}$ & $2 \mathrm{~h}$ post SAH & $5,525,24$ & $24 \mathrm{~h}$ & $\begin{array}{l}\text { BWC (lower } \\
\text { is better); NS (higher } \\
\text { is better) }\end{array}$ \\
\hline Chen et al. (17) & $\begin{array}{l}\text { SD rats, } \\
\text { Male }\end{array}$ & Adult & pentobarbital & i.p. & $\begin{array}{l}\text { Endovascular } \\
\text { perforation }\end{array}$ & $150 \mathrm{mg} / \mathrm{kg}$ & $150 \mathrm{mg} / \mathrm{kg}$ & $2 \mathrm{~h}$ post SAH & $6,624,24$ & $24 \mathrm{~h}$ & $\begin{array}{l}\text { BWC (lower } \\
\text { is better); NS (higher } \\
\text { is better) }\end{array}$ \\
\hline Chen et al. (27) & $\begin{array}{l}\text { SD rats, } \\
\text { Male }\end{array}$ & Adult & pentobarbital & i.p. & $\begin{array}{l}\text { Endovascular } \\
\text { perforation }\end{array}$ & $150 \mathrm{mg} / \mathrm{kg}$ & $150 \mathrm{mg} / \mathrm{kg}$ & $2 \mathrm{~h}$ post SAH & 20,17 & $24 \mathrm{~h}$ & NS (higher is better) \\
\hline Dong et al. (28) & $\begin{array}{l}\text { C57 mice, } \\
\text { Male }\end{array}$ & Adult & isoflurane & i.p. & $\begin{array}{l}\text { Endovascular } \\
\text { perforation }\end{array}$ & $150 \mathrm{mg} / \mathrm{kg}$ & $150 \mathrm{mg} / \mathrm{kg}$ & $2 \mathrm{~h}$ post SAH & $6,639,37$ & $24 \mathrm{~h}$ & $\begin{array}{l}\text { BWC (lower } \\
\text { is better) NS (higher } \\
\text { is better) }\end{array}$ \\
\hline Zhao et al. (29) & $\begin{array}{l}\text { C57 mice, } \\
\text { Male }\end{array}$ & Adult & pentobarbital & i.p. & $\begin{array}{l}\text { Endovascular } \\
\text { perforation }\end{array}$ & $150 \mathrm{mg} / \mathrm{kg}$ & $300 \mathrm{mg} / \mathrm{kg}$ & $\begin{array}{l}2 \text { and } 12 \mathrm{~h} \text { post } \\
\text { SAH }\end{array}$ & 6.6 & $24 \mathrm{~h}$ & $\begin{array}{l}\text { BWC (lower } \\
\text { is better); NS (higher } \\
\text { is better) }\end{array}$ \\
\hline Yu (30) & $\begin{array}{l}\text { SD rats, } \\
\text { Male }\end{array}$ & Adult & pentobarbital & i.p. & $\begin{array}{l}\text { Endovascular } \\
\text { perforation }\end{array}$ & $150 \mathrm{mg} / \mathrm{kg}$ & $450 \mathrm{mg} / \mathrm{kg}$ & $\begin{array}{l}2,24 \text {, and } 48 \mathrm{~h} \\
\text { post SAH }\end{array}$ & 26,30 & $72 \mathrm{~h}$ & NS (higher is better) \\
\hline Yu (30) & $\begin{array}{l}\text { SD rats, } \\
\text { Male }\end{array}$ & Adult & pentobarbital & i.p. & $\begin{array}{l}\text { Endovascular } \\
\text { perforation }\end{array}$ & $150 \mathrm{mg} / \mathrm{kg}$ & $150 \mathrm{mg} / \mathrm{kg}$ & $2 \mathrm{~h}$ post SAH & $6,624,24$ & $24 \mathrm{~h}$ & $\begin{array}{l}\text { BWC (lower } \\
\text { is better); NS (higher } \\
\text { is better) }\end{array}$ \\
\hline Cao et al. (31) & $\begin{array}{l}\text { SD rats, } \\
\text { Male }\end{array}$ & Adult & pentobarbital & i.p. & $\begin{array}{l}\text { Endovascular } \\
\text { perforation }\end{array}$ & $150 \mathrm{mg} / \mathrm{kg}$ & $150 \mathrm{mg} / \mathrm{kg}$ & $2 \mathrm{~h}$ post SAH & $6,624,24$ & $24 \mathrm{~h}$ & $\begin{array}{l}\text { BWC (lower } \\
\text { is better); NS (higher } \\
\text { is better) }\end{array}$ \\
\hline Shi et al. (32) & $\begin{array}{l}\text { SD rats, } \\
\text { Male }\end{array}$ & Adult & pentobarbital & i.p. & $\begin{array}{l}\text { Endovascular } \\
\text { perforation }\end{array}$ & $5 \mathrm{mg} / \mathrm{kg} ; 10 \mathrm{mg} / \mathrm{kg}$ & $5 \mathrm{mg} / \mathrm{kg} ; 10 \mathrm{mg} / \mathrm{kg}$ & $2 \mathrm{~h}$ post SAH & $6,618,18$ & $24 \mathrm{~h}$ & $\begin{array}{l}\text { BWC (lower } \\
\text { is better); NS (higher } \\
\text { is better) }\end{array}$ \\
\hline Shi et al. (32) & $\begin{array}{l}\text { SD rats, } \\
\text { Male }\end{array}$ & Adult & pentobarbital & i.p. & $\begin{array}{l}\text { Endovascular } \\
\text { perforation }\end{array}$ & 10 mg/kg & $10 \mathrm{mg} / \mathrm{kg}$ & $2 \mathrm{~h}$ post SAH & 12,12 & $24 \mathrm{~h}$ & NS (higher is better) \\
\hline
\end{tabular}

SAH, subarachnoid hemorrhage; Tre, treated; Con, control; i.p., intraperitoneal; BWC, brain water content; NS, Neurobehavioral score; NR, not reported. 
A

Study

ID

Ayer $(2008)$

Ayer (2008)

Ayer (2008)

Ayer (2008)

Mehmet (2009)

Wang (2012)

Chen1 (2014)

Chen1 (2014)

Chen1 (2014)

Chent (2014)

Chen2 (2014)

Dong (2016)

Zhao (2017)

Zhao (2017)

Zhao (2017)

Zhao (2017)

Yu (2017)

Cso (2017)

Shi (2018)

Shi (2018)

Shi (2018)

Shi (2018)

Shi (2018)

Shi (2018)

Shi (2018)

Shi (2018)

Overall (1-squared $=55.4 \%, \rho=0.000$ )

NOTE: Weights are from random effects anplysi

$$
-8.99
$$

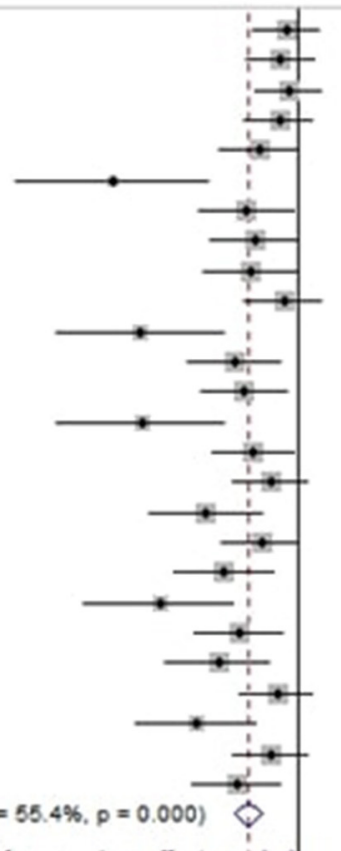

0
$\%$

Weight

$-0.33(-1.43,0.77) 4.96$

$-0.54(-1.06,0.57) 4.91$

$-0.28(-1.37,0.82) 4.97$

$-0.58(-1.70,0.55) \quad 4.90$

$-1.23(-2.51,0.05) 4.45$

$-5.89(-8.99,-2.80) 1.53$

$-1.62(-3.15,-0.08) 3.79$

$-1.37(-2.83,0.09) 3.98$

$-1.49(-2.99,0.00) 3.89$

$-0.43(-1.70,0.83) 4.50$

$-4.99(-7.68,-2.31) 1.90$

$-2.03(-3.53,-0.52) 3.87$

$-1.68(-3.08 .-0.28) 4.14$

$-4.98(-7.67,-2.30) 1.91$

$-1.39(-2.71,-0.07) 4.34$

$-0.85(-2.06,0.35) 4.60$

$-2.92(-4.74,-1.10) 3.18$

$-1.17(-2.43,0.10) 4.49$

$-2.34(-3.95,-0.73) 3.62$

$-4.39(-6.82,-1.97) 2.20$

$-1.83(-3.28,-0.39) 4.02$

$-2.53(-4.20,-0.85) 3.48$

$-0.65(-1.83,0.52) 4.74$

$-3.21(-5.15,-1.28) 2.96$

$-0.84(-2.05,0.36) 4.60$

$-1.93(-3.40,-0.45) 3.95$

$-1.59(-2.02,-1.16) 100.00$

B

\section{Study}

10

\section{Mehmet (2009)}

Cai (2013)

Chent (2014)

Chen2 (2014)

Chen (2015)

Dong (2016)

Zhao (2017)

Yu (2017)

Yu (2017)

CaO (2017)

Shi (2018)

Shi (2018)

Shi (2018)

Overall (1-squared $=81.8 \%, \rho=0.000$ )

NOTE: Weights are from random effects analysis

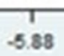

*

SMO $(95 \% \mathrm{Cr}) \quad$ Weight

$2.50(0.83,4.16) \quad 5.60$

$1.58(0.41,2.74) \quad 7.18$

$2.03(1.33,2.73) \quad 8.09$

$1.33(0.70 .1 .90) \quad 8.90$

$3.28(2.26 .4 .30) \quad 7.00$

4.95 (4.03.5.88) $\quad 7.90$

$3.47(1.43,5.50) \quad 4.00$

$3.01(223,3.79) \quad 8.45$

$2.70(1.95,3.57) \quad 8.30$

$2.84(1.85,3.43) \quad 8.42$

$2.85(1.89 .3 .80) \quad 7.88$

$2.05(1.73,3.57) \quad 8.00$

$0.79(-0.05,1.02) \quad 8.27$

$2.50(1.97 .3 .16) \quad 100.00$

FIGURE 2 | Effect sizes of included comparisons. A forest plot shows mean effect size and 95\% Cl for (A) brain water content and (B) neurobehavioral outcomes. 
TABLE 2 | Methodological quality of 12 studies included in the meta-analysis.

\begin{tabular}{|c|c|c|c|c|c|c|c|c|c|c|c|}
\hline Study & (1) & (2) & (3) & (4) & (5) & (6) & (7) & (8) & (9) & (10) & Total \\
\hline Ayer et al. (22) & $\sqrt{ }$ & $\sqrt{ }$ & & & & & & & & $\sqrt{ }$ & 3 \\
\hline Ersahin et al. (23) & $\sqrt{ }$ & $\sqrt{ }$ & & & $\sqrt{ }$ & & & & $\sqrt{ }$ & $\sqrt{ }$ & 5 \\
\hline Wang et al. (24) & $\sqrt{ }$ & $\sqrt{ }$ & & & $\sqrt{ }$ & & & & $\sqrt{ }$ & $\sqrt{ }$ & 5 \\
\hline Cai et al. (25) & $\sqrt{ }$ & $\sqrt{ }$ & $\sqrt{ }$ & & $\sqrt{ }$ & & & & $\sqrt{ }$ & $\sqrt{ }$ & 6 \\
\hline Chen et al. (26) & $\sqrt{ }$ & $\sqrt{ }$ & $\sqrt{ }$ & & $\sqrt{ }$ & $\sqrt{ }$ & & & $\sqrt{ }$ & $\sqrt{ }$ & 7 \\
\hline Chen et al. (17) & $\sqrt{ }$ & $\sqrt{ }$ & $\sqrt{ }$ & & $\sqrt{ }$ & $\sqrt{ }$ & & & $\sqrt{ }$ & $\sqrt{ }$ & 7 \\
\hline Chen et al. (27) & $\sqrt{ }$ & & $\sqrt{ }$ & & $\sqrt{ }$ & $\sqrt{ }$ & & & $\sqrt{ }$ & $\sqrt{ }$ & 6 \\
\hline Dong et al. (28) & $\sqrt{ }$ & $\sqrt{ }$ & $\sqrt{ }$ & & $\sqrt{ }$ & $\sqrt{ }$ & & & $\sqrt{ }$ & $\sqrt{ }$ & 7 \\
\hline Zhao et al. (29) & $\sqrt{ }$ & $\sqrt{ }$ & $\sqrt{ }$ & & $\sqrt{ }$ & $\sqrt{ }$ & & & $\sqrt{ }$ & $\sqrt{ }$ & 7 \\
\hline Yu (30) & $\sqrt{ }$ & $\sqrt{ }$ & $\sqrt{ }$ & & & $\sqrt{ }$ & & & $\sqrt{ }$ & $\sqrt{ }$ & 6 \\
\hline Cao et al. (31) & $\sqrt{ }$ & & $\sqrt{ }$ & & $\sqrt{ }$ & $\sqrt{ }$ & & & $\sqrt{ }$ & $\sqrt{ }$ & 6 \\
\hline Shi et al. (32) & $\sqrt{ }$ & $\sqrt{ }$ & $\sqrt{ }$ & & $\sqrt{ }$ & $\sqrt{ }$ & & & $\sqrt{ }$ & $\sqrt{ }$ & 7 \\
\hline
\end{tabular}

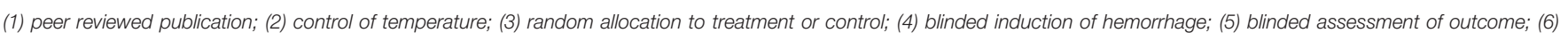

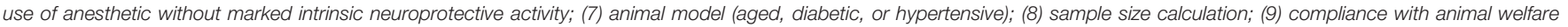
regulations; and (10) statement of potential conflict of interests.

statistically significant heterogeneity $\left(\mathrm{Q}=56.02, I^{2}=55.4 \%, \mathrm{df}\right.$ $=25, p<0.001$ ).

On the other hand, the global estimate of the efficacy of melatonin in improving the NS outcomes was 2.56 (95\% CI: 1.97, 3.16; $p<0.0001,10$ studies, 13 comparisons, Figure 2B). The heterogeneity of NS outcomes among comparisons was also statistically significant $\left(\mathrm{Q}=66.08, I^{2}=81.8 \%\right.$, df $=12, p<$ $0.001)$. Therefore, further subgroup analysis was performed.

\section{Sensitivity Analysis}

To evaluate the stability of the results, we performed a sensitivity analysis through the sequential omission of each study. For the pooled SMD, neither brain water content nor neurobehavioral outcomes were significantly affected by any study (Figures 3A,B).

\section{Publication Bias}

Visual inspection of the funnel plot suggested conspicuous publication bias for the brain water content (Figure 4A), and the results of the Egger test suggested the same comment ( $p$ $<0.001)$. The trim-and-fill analysis was used to estimate the missing studies (SMD: $-1.59,95 \% \mathrm{CI}:-2.02,-1.16 ; p<0.001$ ), indicating no "missing" studies.

For the neurobehavioral outcomes, the funnel plot was approximately symmetrical (Figure 4B) and the Egger test indicated no significant publication bias $(\mathrm{p}=0.305)$. However, two theoretically missing comparisons of neurobehavioral outcomes were predicted (Figure 4C).

\section{Subgroup analysis}

We performed a subgroup analysis of brain water content and neurobehavioral score; the details of the data are shown in Tables 3, 4.

For brain water content, we stratified the data by study quality; the high-quality studies showed a higher effect size (SMD: $-1.86 ; 95 \%$ CI: $-2.33,-1.40 ; p<0.001$,
Supplementary Figure 1) and the results of the low-quality studies were not statistically significant.

Different SAH induction methods were used in studies, and our subgroup analysis showed that the results of the autogenous blood model (SMD: -3.34 ; 95\% CI: $-7.89,1.21 ; p=0.15$, Supplementary Figure 2) were more effective than those of the endovascular perforation model with no statistical significance.

Concerning the anesthesia drug, the studies that used isoflurane exhibited a higher effect size than others (SMD: -2.03 ; 95\% CI: $-3.53,-0.52 ; p<0.001$, Supplementary Figure 3). However, the small sample size makes the assessments less reliable.

The dosage of melatonin ranged from 5 to $150 \mathrm{mg} / \mathrm{kg}$, and the greatest effect was exerted at the dosage of 10 $\mathrm{mg} / \mathrm{kg}$ (SMD: $-2.38 ; 95 \%$ CI: $-3.35,-1.41 ; p<0.001$, Supplementary Figure 4). Further, we found the repeated melatonin dosage showed a greater effect size than a single dosage (SMD: -2.11 ; 95\% CI: $-3.27,-0.95 ; p<0.001$, Supplementary Figure 5).

For the neurobehavioral score, our subgroup analysis showed that the endovascular perforation model exhibited a higher effect size than the autogenous blood model (SMD: 2.65; 95\% CI: 1.99, $3.31 ; p<0.001$, Supplementary Figure 6).

The studies that used isoflurane showed a higher effect size than other anesthetics (SMD: 4.95; 95\% CI: 4.03, 5.88; $p<0.001$, Supplementary Figure 7), which was consistent with the results of the brain water content.

The dosage of melatonin ranged from 5 to $150 \mathrm{mg} / \mathrm{kg}$, and in a result different from that of brain water content, the studies using a dosage of $150 \mathrm{mg} / \mathrm{kg}$ exhibited the highest effect size (SMD: 2.39; 95\% CI: 1.86, 2.92; $p<0.001$, Supplementary Figure 8).

Besides, we stratified the data by dose administration, and there was no significant effect size difference in neuroprotective effects for both the doses administered (Supplementary Figure 9). 


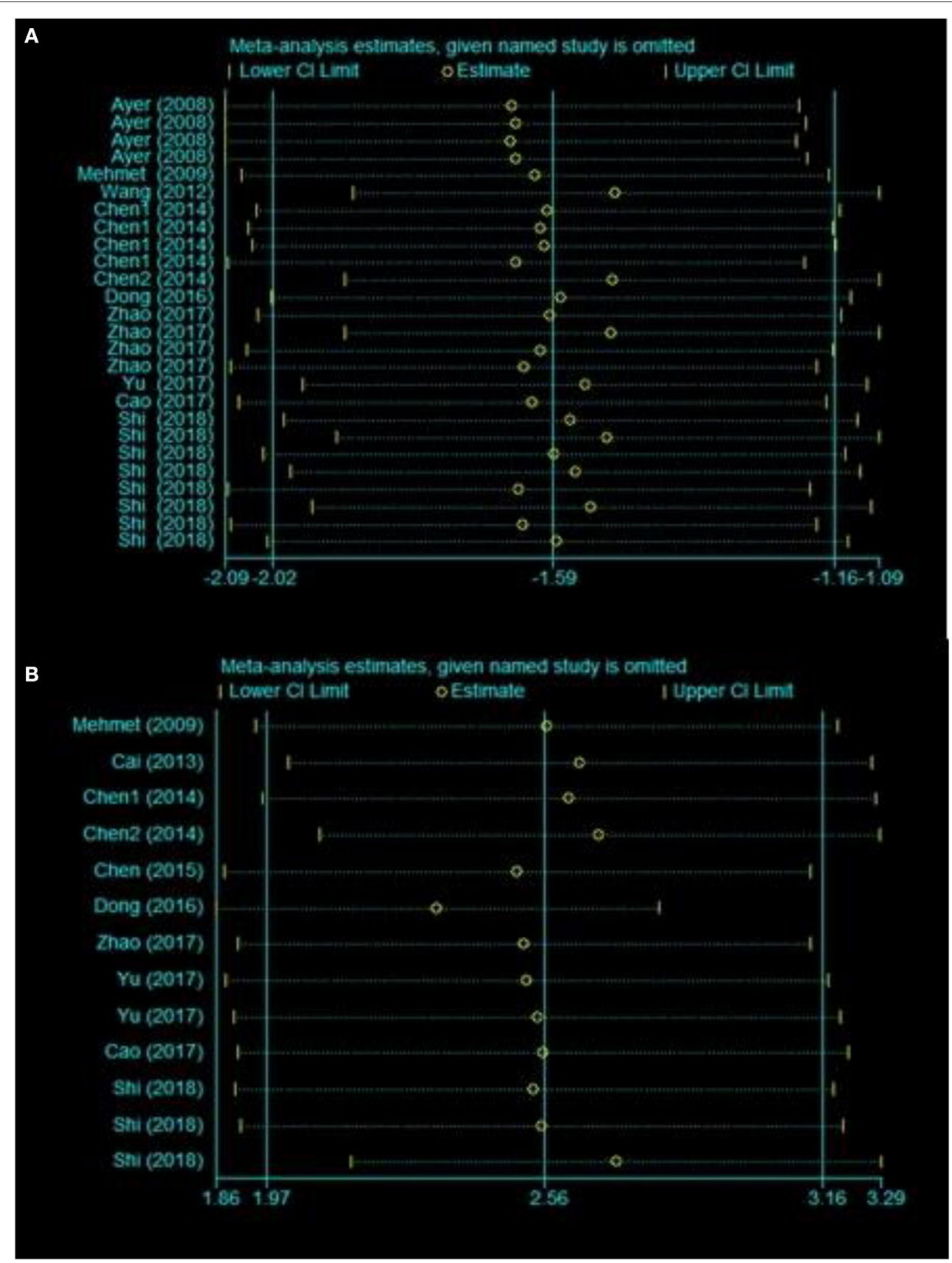

FIGURE 3 | Sensitivity analysis for the included comparisons. Figures shows mean effect size and 95\% Cl for (A) brain water content and (B) neurobehavioral outcomes.

\section{DISCUSSION}

\section{Summary of Evidence}

Although there have been related systematic reviews on melatonin in experimental cerebral ischemia (33) and traumatic brain injury (34), as far as we know, this is the first systematic review and meta-analysis of melatonin in an animal model with SAH. The outcomes of our systematic review indicate that melatonin shows significant neuroprotective effects in reducing brain water content [SMD: -1.59 (95\% CI: $-2.02,-1.16)$ ] and improving neurobehavioral outcomes [SMD: 2.56 (95\% CI: 1.97, 3.16)]. These findings signify that melatonin can be used as a potential treatment for clinical SAH. However, due to the limited number of studies and poor literature quality, more studies are needed to prove the neuroprotective effect of melatonin in experimental SAH. 


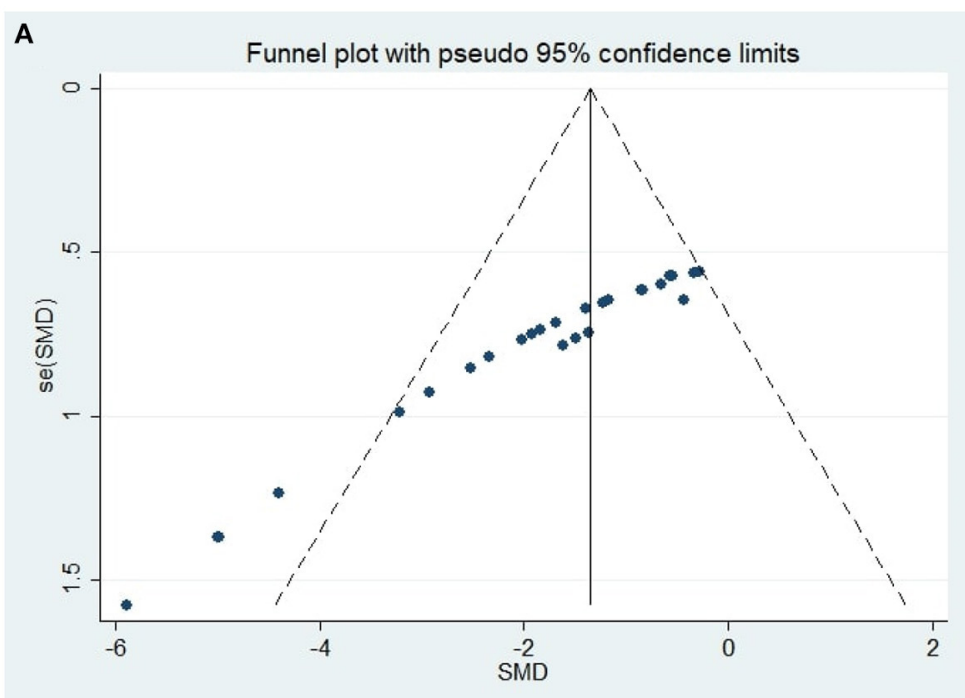

B

Funnel plot with pseudo $95 \%$ confidence limits
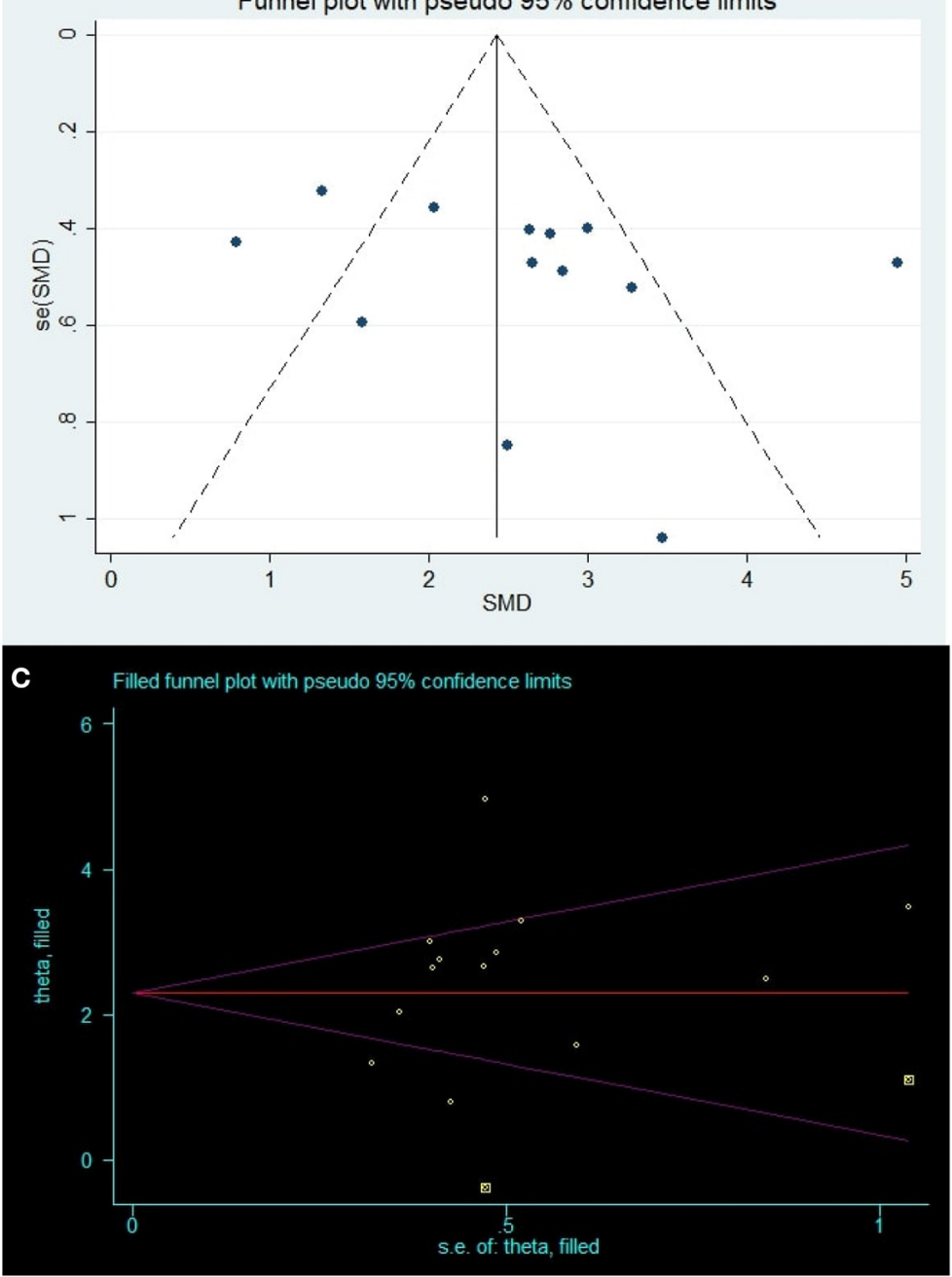

FIGURE 4 | Publication bias. Funnel plots for (A) brain water content and (B) neurobehavioral outcomes; Yellow points in (C) represent theoretically missing comparisons identified using the trim-and-fill method. 
TABLE 3 | Stratified meta-analysis of heterogeneity on brain water content.

\begin{tabular}{|c|c|c|c|c|c|}
\hline \multirow[t]{2}{*}{ Subgroup analysis } & \multirow[t]{2}{*}{ No. of studies } & \multirow[t]{2}{*}{ SMD $(95 \% \mathrm{Cl})$} & \multicolumn{3}{|c|}{ Heterogeneity test } \\
\hline & & & $\mathbf{Q}$ & $I^{2}$ & $p$ \\
\hline \multicolumn{6}{|l|}{ 1.1 Study quality } \\
\hline High & 22 & $-1.86(-2.33,-1.40)$ & 41.65 & $50 \%$ & 0.005 \\
\hline Low & 4 & $-0.43(-0.98,0.13)$ & 0.21 & $0 \%$ & 0.98 \\
\hline \multicolumn{6}{|c|}{ 1.2 Method to induce SAH } \\
\hline Endovascular perforation & 24 & $-1.52(-1.94,-1.09)$ & 47.63 & $52 \%$ & 0.002 \\
\hline Autogenous blood & 2 & $-3.34(-7.89,1.21)$ & 7.46 & $87 \%$ & 0.006 \\
\hline \multicolumn{6}{|l|}{ 1.3 Anesthetic drugs } \\
\hline Pentobarbital & 19 & $-1.79(-2.28,-1.31)$ & 33.74 & $47 \%$ & 0.01 \\
\hline Ketamine & 6 & $-0.89(-1.72,-0.05)$ & 12.63 & $60 \%$ & 0.03 \\
\hline Isoflurane & 1 & $-2.03(-3.53,-0.52)$ & & & \\
\hline \multicolumn{6}{|l|}{ 1.4 Dosage of melatonin } \\
\hline $5 \mathrm{mg} / \mathrm{kg}$ & 4 & $-1.28(-2.04,-0.53)$ & 3.83 & $22 \%$ & 0.28 \\
\hline 10 mg/kg & 5 & $-2.38(-3.35,-1.41)$ & 6.68 & $40 \%$ & 0.15 \\
\hline 150 mg/kg & 17 & $-1.46(-2.01,-0.91)$ & 39.11 & $59 \%$ & 0.001 \\
\hline \multicolumn{6}{|l|}{ 1.5 Dose administration } \\
\hline Single dose & 20 & $-1.48(-1.94,-1.01)$ & 39.55 & $52 \%$ & 0.004 \\
\hline Repeat dose & 6 & $-2.11(-3.27,-0.95)$ & 15.41 & $68 \%$ & 0.009 \\
\hline
\end{tabular}

TABLE 4 | Stratified meta-analysis of heterogeneity on neurobehavioral score.

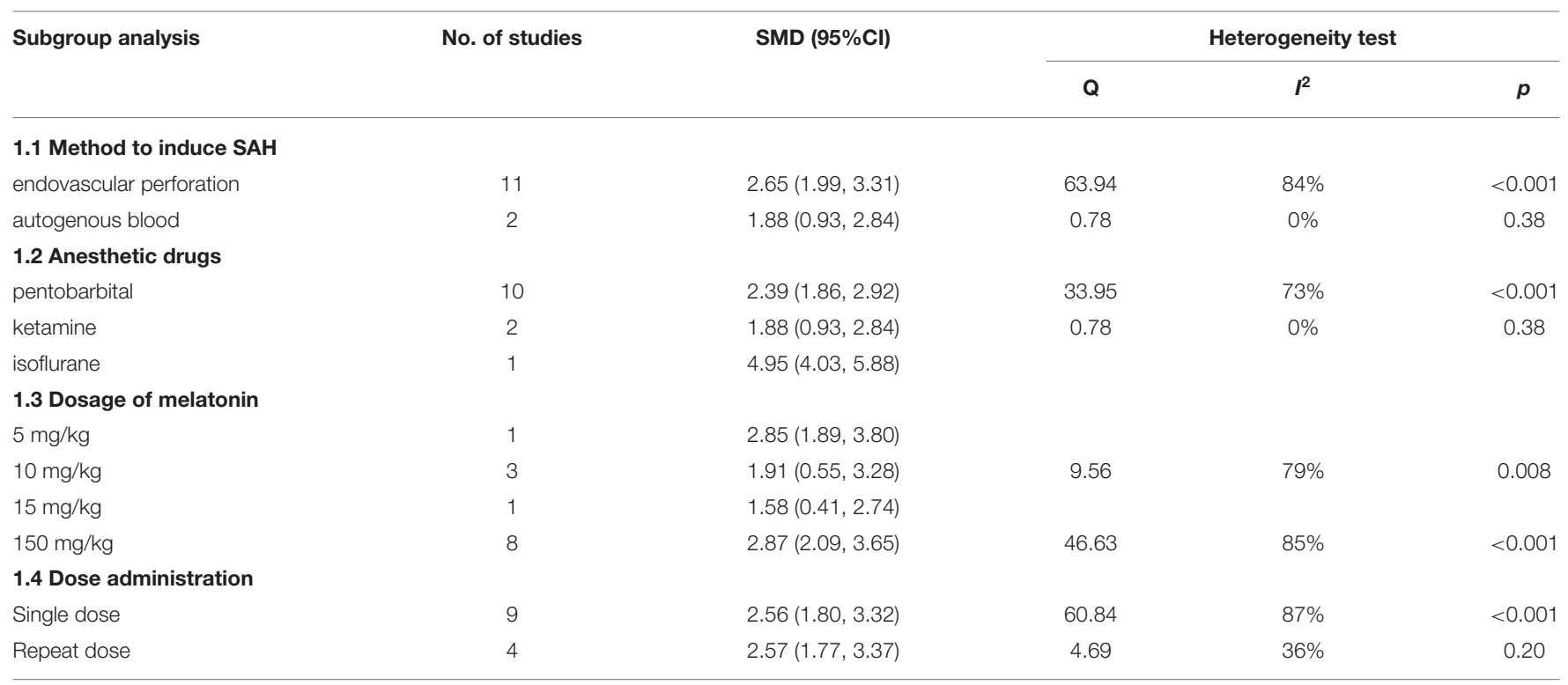

\section{Interpretation of Subgroup Analysis}

As a basic type of stroke, SAH has received more and more attention because of its high morbidity and mortality. The earlystage-damage of SAH is related mainly to the impaired brain autoregulation. The subsequent cerebral vasospasm/delayed cerebral ischemia significantly affects patients' prognosis (35, 36). As a non-toxic antioxidant, melatonin has attracted much attention because of good tolerance and high penetration level through the blood-brain barrier. It is generally believed that melatonin can exert its neuroprotective effects on SAH in many aspects. On the one hand, melatonin can enhance earlystage brain autoregulation through inhibiting sympathetic nerve activity, mediating myogenic response, alleviating hypoxemia, and regulating metabolism. On the other hand, melatonin plays an antioxidant role to protect endothelial cell function, increase nitric oxide availability, and relieve vasospasm/delayed cerebral ischemia (37). In this study, melatonin presented significant effects on improving neurological score and ameliorating 
cerebral edema. Nevertheless, due to obvious heterogeneity of the result, the next step of subgroup analysis was performed. The results show that methodological differences including modeling methods, anesthetic drugs, the dosage of melatonin, and dose administration have contributed to the heterogeneity of the results.

For brain water content, the results of the subgroup analysis showed that melatonin has a higher efficacy in the high study quality group. Previous studies have found that research quality has a significant impact on the outcome; it is easier to exaggerate the effect size in low-quality studies $(38,39)$. However, some studies believe that the research quality has no significant influence on the effect size (40). In our meta-analysis, although the high-quality studies show better results compared with the low-quality studies, more research is needed to get a positive result, considering the limited number of low-quality studies.

There is no clear relationship between the modeling methods and effect size. For brain water content, the autologous blood injection model achieved a better result with no statistical significance, but the opposite results were achieved in the neurobehavioral score group, wherein the endovascular perforation model was more effective. At present, no animal model can simulate the physiological conditions of human subarachnoid hemorrhage. For the autologous blood injection model, the volume of blood and the injection site varied in different studies. For the endovascular perforation model, the sutures used for puncture were different. Besides, the types of rats and animal weights included in our research were also not the same. It can be considered that the lack of a unified SAH animal model is the main reason for the differentiated results $(41,42)$.

Our results showed that isoflurane achieved the best effect in both the brain water content and neurobehavioral score groups, but the results are less reliable considering the small sample size. In fact, the neuroprotective effects of phenobarbital, isoflurane, and ketamine anesthetics have been widely reported (43-45), and the specific mechanism may be related to the anesthetic's inhibition of GABA-a inhibitory and NMDA excitatory receptors $(46,47)$. Although the effect of anesthetics on the pathophysiological process of subarachnoid hemorrhage is still unclear, the potential neuroprotective effect cannot be ignored when the results are interpreted.

The dosage of melatonin ranged from 5 to $150 \mathrm{mg} / \mathrm{kg}$. In the brain water content group, $10 \mathrm{mg} / \mathrm{kg}$ achieved the greatest effect size, while in the neurobehavioral score group, $150 \mathrm{mg} / \mathrm{kg}$ had the best effect size. It is difficult to conclude at what dosage the drug achieves the best effect due to the wide range of drugs. Furthermore, the results showed that the repeated doses had greater efficacy than the single doses, which indicated a short duration of action after single-dose treatment. This may be further related to the fact that the metabolic half-life of melatonin in rats by oral or intravenous injection was $<20 \mathrm{~min}$ (48).

\section{Advantages and Limitation}

Our study made a lot of effort to obtain relatively objective results. First, this meta-analysis collected the most comprehensive reports in this field possible, representing the most complete analysis of the use of melatonin in SAH animal models.
Second, two experienced researchers independently evaluated and extracted all the data studies included to reduce potential publication bias. Finally, our results showed that both the behavior and pathological results of SAH animal models treated with melatonin showed significant improvement, indicating melatonin can probably be used as a new treatment strategy in the treatment of clinical SAH patients.

Although this study achieved positive results, the possible shortcomings should not be ignored. First, our research only includes the available data; some negative results are less likely to have been published. The cut-and-fill method shows two theoretically missing studies on the neurobehavioral score group. Therefore, this meta-analysis may have exaggerated the effect size. Second, the studies we included were highly heterogeneous with an $I^{2}$ of 55.4 and $81.8 \%$, which may be related to animal species, study quality, modeling methods, anesthetics, drug dosage, and dose administration; therefore, a further subgroup analysis was carried out. However, due to the limited sample size of each subgroup and insufficient statistical power, the differences between some subgroups were not obvious. Third, our study still lacks the findings of the effect of melatonin in SAH rat models with specific types of diseases (such as diabetes and hypertension). The clinical patients are more likely to present with different types of underlying diseases, so there is still a lot of work to be done in clinical translation.

\section{CONCLUSION}

Our research shows that melatonin can significantly improve the behavior and pathological results of SAH animal models. However, due to the small number of studies included in this meta-analysis, the experimental design and experimental method limitations should be considered when interpreting the results. Significant clinical and animal studies are still required to evaluate whether melatonin can be used in the adjuvant treatment of clinical SAH patients.

\section{DATA AVAILABILITY STATEMENT}

The original contributions presented in the study are included in the article/Supplementary Material, further inquiries can be directed to the corresponding author/s.

\section{AUTHOR CONTRIBUTIONS}

$\mathrm{ZH}$ : study concept and design. $\mathrm{XH}$ and YZ: acquisition of data. CC, CP, and FZ: analysis and interpretation of data. XH and YZ: drafting of the manuscript. CC and $\mathrm{ZH}$ : revision of manuscript. CC: supervision of work. All authors read and approved the final manuscript.

\section{SUPPLEMENTARY MATERIAL}

The Supplementary Material for this article can be found online at: https://www.frontiersin.org/articles/10.3389/fneur. 2021.685731/full\#supplementary-material 


\section{REFERENCES}

1. Wang W, Jiang B, Sun H, Ru X, Sun D, Wang L, et al. Prevalence, incidence, and mortality of stroke in china: results from a nationwide population-based survey of 480687 adults. Circulation. (2017) 135:759-71. doi: 10.1161/CIRCULATIONAHA.116.025250

2. van Gijn J, Kerr RS, Rinkel GJ. Subarachnoid haemorrhage. Lancet. (2007) 369:306-18. doi: 10.1016/S0140-6736(07)60153-6

3. Gareev I, Beylerli O, Aliev G, Pavlov V, Izmailov A, Zhang Y, et al. The role of long non-coding RNAs in intracranial aneurysms and subarachnoid hemorrhage. Life. (2020) 10:155. doi: 10.3390/life10090155

4. Molyneux AJ, Kerr RS, Yu LM, Clarke M, Sneade M, Yarnold JA, et al. International subarachnoid aneurysm trial (ISAT) of neurosurgical clipping versus endovascular coiling in 2143 patients with ruptured intracranial aneurysms: a randomised comparison of effects on survival, dependency, seizures, rebleeding, subgroups, and aneurysm occlusion. Lancet. (2005) 366:809-17. doi: 10.1016/S0140-6736(05)67214-5

5. Park S, Yamaguchi M, Zhou C, Calvert JW, Tang J, Zhang JH. Neurovascular protection reduces early brain injury after subarachnoid hemorrhage. Stroke. (2004) 35:2412-7. doi: 10.1161/01.STR.0000141162.29864.e9

6. Provencio JJ, Altay T, Smithason S, Moore SK, Ransohoff RM. Depletion of Ly6G/C $(+)$ cells ameliorates delayed cerebral vasospasm in subarachnoid hemorrhage. J Neuroimmunol. (2011) 232:94-100. doi: 10.1016/j.jneuroim.2010.10.016

7. Kwon MS, Woo SK, Kurland DB, Yoon SH, Palmer AF, Banerjee U, et al. Methemoglobin is an endogenous toll-like receptor 4 ligandrelevance to subarachnoid hemorrhage. Int J Mol Sci. (2015) 16:5028-46. doi: 10.3390/ijms16035028

8. Fujimoto M, Shiba M, Kawakita F, Liu L, Shimojo N, Imanaka-Yoshida $\mathrm{K}$, et al. Effects of tenascin-c knockout on cerebral vasospasm after experimental subarachnoid hemorrhage in mice. Mol Neurobiol. (2018) 55:1951-8. doi: 10.1007/s12035-017-0466-x

9. Gu X, Zheng C, Zheng Q, Chen S, Li W, Shang Z, et al. Salvianolic acid A attenuates early brain injury after subarachnoid hemorrhage in rats by regulating ERK/P38/Nrf2 signaling. Am J Transl Res. (2017) 9:5643-52.

10. Shao A, Wang Z, Wu H, Dong X, Li Y, Tu S, et al. Enhancement of autophagy by histone deacetylase inhibitor trichostatin a ameliorates neuronal apoptosis after subarachnoid hemorrhage in rats. Mol Neurobiol. (2016) 53:18-27. doi: 10.1007/s12035-014-8986-0

11. Jing CH, Wang L, Liu PP, Wu C, Ruan D, Chen G. Autophagy activation is associated with neuroprotection against apoptosis via a mitochondrial pathway in a rat model of subarachnoid hemorrhage. Neuroscience. (2012) 213:144-53. doi: 10.1016/j.neuroscience.2012.03.055

12. Carrillo-Vico A, Calvo JR, Abreu P, Lardone PJ, García-Mauriño S, Reiter RJ, et al. Evidence of melatonin synthesis by human lymphocytes and its physiological significance: possible role as intracrine, autocrine, and/or paracrine substance. FASEB J. (2004) 18:537-9. doi: 10.1096/fj.03-0694fje

13. Cheung RT. The utility of melatonin in reducing cerebral damage resulting from ischemia and reperfusion. J Pineal Res. (2003) 34:153-60. doi: 10.1034/j.1600-079x.2003.00034.x

14. Esposito E, Paterniti I, Mazzon E, Bramanti P, Cuzzocrea S. Melatonin reduces hyperalgesia associated with inflammation. J Pineal Res. (2010) 49:321-31. doi: 10.1111/j.1600-079X.2010.00796.x

15. Caballero B, Vega-Naredo I, Sierra V, DeGonzalo-Calvo D, MedranoCampillo P, Guerrero JM, et al. Autophagy upregulation and loss of NFkappaB in oxidative stress-related immunodeficient SAMP8 mice. Mech Ageing Dev. (2009) 130:722-30. doi: 10.1016/j.mad.2009.09.001

16. Yang S, Tang W, He Y, Wen L, Sun B, Li S. Long non-coding RNA and microRNA-675/let-7a mediates the protective effect of melatonin against early brain injury after subarachnoid hemorrhage via targeting TP53 and neural growth factor. Cell Death Dis. (2018) 9:99. doi: 10.1038/s41419-017-0155-8

17. Chen J, Wang L, Wu C, Hu Q, Gu C, Yan F, et al. Melatonin-enhanced autophagy protects against neural apoptosis via a mitochondrial pathway in early brain injury following a subarachnoid hemorrhage. J Pineal Res. (2014) 56:12-9. doi: 10.1111/jpi.12086

18. Geyik M, Erkutlu I, Geyik S, Alptekin M, Gezgin I, Gok A. Paradoxical morphometric and antioxidative effects of melatonin on vasospasm in experimental subarachnoid hemorrhage. J Neurol Sci. (2015) 32:756-66.
19. Macleod MR, O'Collins T, Howells DW, Donnan GA. Pooling of animal experimental data reveals influence of study design and publication bias. Stroke. (2004) 35:1203-8. doi: 10.1161/01.STR.0000125719.25853.20

20. DerSimonian R, Laird N. Meta-analysis in clinical trials revisited. Contemp Clin Trials. (2015) 45:139-45. doi: 10.1016/j.cct.2015.09.002

21. Song F, Gilbody S. Bias in meta-analysis detected by a simple, graphical test. Increase in studies of publication bias coincided with increasing use of meta-analysis. BMJ. (1998) 316:471.

22. Ayer RE, Sugawara T, Zhang JH. Effects of melatonin in early brain injury following subarachnoid hemorrhage. Acta Neurochir Suppl. (2008) 102:32730. doi: 10.1007/978-3-211-85578-2_62

23. Ersahin M, Toklu HZ, Cetinel S, Yüksel M, Yegen BC, Sener G. Melatonin reduces experimental subarachnoid hemorrhage-induced oxidative brain damage and neurological symptoms. J Pineal Res. (2009) 46:324-32. doi: 10.1111/j.1600-079X.2009.00664.x

24. Wang Z, Ma C, Meng CJ, Zhu GQ, Sun XB, Huo L, et al. Melatonin activates the Nrf2-ARE pathway when it protects against early brain injury in a subarachnoid hemorrhage model. J Pineal Res. (2012) 53:129-37. doi: 10.1111/j.1600-079X.2012.00978.x

25. Cai J, He C, Chen L, Han T, Huang S, Huang Y, et al. Melatonin mitigate cerebral vasospasm after experimental subarachnoid hemorrhage: a study of synchrotron radiation angiography. J Instrument. (2013) 8:C06004. doi: 10.1088/1748-0221/8/06/C06004

26. Chen J, Chen G, Li J, Qian C, Mo H, Gu C, et al. Melatonin attenuates inflammatory response-induced brain edema in early brain injury following a subarachnoid hemorrhage: a possible role for the regulation of proinflammatory cytokines. J Pineal Res. (2014) 57:340-7. doi: 10.1111/jpi.12173

27. Chen J, Qian C, Duan H, Cao S, Yu X, Li J, et al. Melatonin attenuates neurogenic pulmonary edema via the regulation of inflammation and apoptosis after subarachnoid hemorrhage in rats. J Pineal Res. (2015) 59:46977. doi: 10.1111/jpi.12278

28. Dong Y, Fan C, Hu W, Jiang S, Ma Z, Yan X, et al. Melatonin attenuated early brain injury induced by subarachnoid hemorrhage via regulating NLRP3 inflammasome and apoptosis signaling. J Pineal Res. (2016) 60:253-62. doi: 10.1111/jpi.12300

29. Zhao L, Liu H, Yue L, Zhang J, Li X, Wang B, et al. Melatonin attenuates early brain injury via the melatonin receptor/Sirt1/NF- $\kappa \mathrm{B}$ signaling pathway following subarachnoid hemorrhage in mice. Mol Neurobiol. (2017) 54:161221. doi: $10.1007 / \mathrm{s} 12035-016-9776-7$

30. Yu X. 俞晓波, 褪黑素在蛛网膜下腔出血早期中抗坏死性淍亡和抗神炎症 的机制研究(2017) 浙江大学.

31. Cao S, Shrestha S, Li J, Yu X, Chen J, Yan F, et al. Melatonin-mediated mitophagy protects against early brain injury after subarachnoid hemorrhage through inhibition of NLRP3 inflammasome activation. Sci Rep. (2017) 7:2417. doi: 10.1038/s41598-017-02679-z

32. Shi L, Liang F, Zheng J, Zhou K, Chen S, Yu J, et al. Melatonin regulates apoptosis and autophagy via ROS-MST1 pathway in subarachnoid hemorrhage. Front Mol Neurosci. (2018) 11:93. doi: 10.3389/fnmol.2018.00093

33. Macleod MR, O'Collins T, Horky LL, Howells DW, Donnan GA. Systematic review and meta-analysis of the efficacy of melatonin in experimental stroke. J Pineal Res. (2005) 38:35-41. doi: 10.1111/j.1600-079X.2004.00172.x

34. Barlow KM, Esser MJ, Veidt M, Boyd R. Melatonin as a treatment after traumatic brain injury: a systematic review and meta-analysis of the pre-clinical and clinical literature. J Neurotrauma. (2019) 36:523-37. doi: 10.1089/neu.2018.5752

35. Ye ZN, Wu LY, Liu JP, Chen Q, Zhang XS, Lu Y, et al. Inhibition of leukotriene B4 synthesis protects against early brain injury possibly via reducing the neutrophil-generated inflammatory response and oxidative stress after subarachnoid hemorrhage in rats. Behav Brain Res. (2018) 339:1927. doi: 10.1016/j.bbr.2017.11.011

36. Zhang ZY, Sun BL, Yang MF, Li DW, Fang J, Zhang S. Carnosine attenuates early brain injury through its antioxidative and anti-apoptotic effects in a rat experimental subarachnoid hemorrhage model. Cell Mol Neurobiol. (2015) 35:147-57. doi: 10.1007/s10571-014-0150-x

37. Guo ZN, Jin H, Sun H, Zhao Y, Liu J, Ma H, et al. Antioxidant melatonin: potential functions in improving cerebral autoregulation after subarachnoid hemorrhage. Front Physiol. (2018) 9:1146. doi: 10.3389/fphys.2018.01146 
38. Sena E, Wheble P, Sandercock P, Macleod M. Systematic review and metaanalysis of the efficacy of tirilazad in experimental stroke. Stroke. (2007) 38:388-94. doi: 10.1161/01.STR.0000254462.75851.22

39. Lythgoe DJ, Little RA, O'shaughnessy CT, Steward MC - Pharmacol Br J. Effect of U-74006F on oedema and infarct volumes following permanent occlusion of the middle cerebral artery in the rat. Br J Pharmacol. (1990) 100:454.

40. Cui HJ, He HY, Yang AL, Zhou HJ, Wang C, Luo JK, et al. Efficacy of deferoxamine in animal models of intracerebral hemorrhage: a systematic review and stratified meta-analysis. PLoS ONE. (2015) 10:e0127256. doi: 10.1371/journal.pone.0127256

41. Kamp MA, Lieshout JHV, Dibué-Adjei M, Weber JK, Schneider T, Restin $\mathrm{T}$, et al. A systematic and meta-analysis of mortality in experimental mouse models analyzing delayed cerebral ischemia after subarachnoid hemorrhage. Transl Stroke Res. (2017) 8:206-19. doi: 10.1007/s12975-016-0513-3

42. Marbacher S. Animal models for the study of subarachnoid hemorrhage: are we moving towards increased standardization? Transl Stroke Res. (2016) 7:1-2. doi: 10.1007/s12975-015-0442-6

43. Sharma HS, Muresanu DF, Nozari A, Castellani RJ, Dey PK, Wiklund L, et al. Anesthetics influence concussive head injury induced blood-brain barrier breakdown, brain edema formation, cerebral blood flow, serotonin levels, brain pathology and functional outcome. Int Rev Neurobiol. (2019) 146:45-81. doi: 10.1016/bs.irn.2019.06.006

44. Marchesini V, Disma N. Anaesthetic neuroprotection in children: does it exist or is it all just bad? Curr Opin Anaesthesiol. (2019) 32:363-9. doi: 10.1097/ACO.0000000000000723

45. Jiang M, Sun L, Feng DX, Yu ZQ, Gao R, Sun YZ, et al. Neuroprotection provided by isoflurane pre-conditioning and postconditioning. Med Gas Res. (2017) 7:48-55. doi: 10.4103/2045-9912. 202910
46. Olsen RW, Li GD. GABA(A) receptors as molecular targets of general anesthetics: identification of binding sites provides clues to allosteric modulation. Can J Anaesth. (2011) 58:206-15. doi: 10.1007/s12630-010-9429-7

47. Muir WW. NMDA receptor antagonists and pain: ketamine. Vet Clin North Am Equine Pract. (2010) 26:565-78. doi: 10.1016/j.cveq.2010.07.009

48. Yeleswaram K, McLaughlin LG, Knipe JO, Schabdach D. Pharmacokinetics and oral bioavailability of exogenous melatonin in preclinical animal models and clinical implications. J Pineal Res. (1997) 22:45-51. doi: 10.1111/j.1600-079X.1997.tb00302.x

Conflict of Interest: The authors declare that the research was conducted in the absence of any commercial or financial relationships that could be construed as a potential conflict of interest.

Publisher's Note: All claims expressed in this article are solely those of the authors and do not necessarily represent those of their affiliated organizations, or those of the publisher, the editors and the reviewers. Any product that may be evaluated in this article, or claim that may be made by its manufacturer, is not guaranteed or endorsed by the publisher.

Copyright (c) $2021 \mathrm{Hu}, \mathrm{Zhu}$, Zhou, Peng, Hu and Chen. This is an open-access article distributed under the terms of the Creative Commons Attribution License (CC $B Y)$. The use, distribution or reproduction in other forums is permitted, provided the original author(s) and the copyright owner(s) are credited and that the original publication in this journal is cited, in accordance with accepted academic practice. No use, distribution or reproduction is permitted which does not comply with these terms. 